\title{
Introduction
}

Sir Francis Galton, a cousin of Charles Darwin, introduced the twin study in his article "The History of Twins, as a Criterion of the Relative Powers of Nature and Nurture" published in 1875 . The vast majority of the twin pairs he studied appear to have been identical twins, and he had no possibility to quantify the effect of heredity and environmental factors by comparing identical and fraternal twins. Still the astonishing similarity between the co-twins he observed led him to boldly conclude that "there is no escape from the conclusion that nature prevails enormously over nurture".

It should take fifty years from Galton's study to the first "classical" twin study to take place. It may now seem surprising that Galton's study did not inspire others to study twins. The time for the first classical twin studies to appear is not coincidental, however. In the 1920s new biological evidence showed that twins were either from the same egg or from two separately fertilized eggs, implying that the classification into identical and fraternal twins were now scientifically grounded. The first authors who actually compared the correlations of identical and fraternal twins, were Gladys Tallman and Alex Wingfield, both in 1928, and both studying intelligence.

During the next 50 years after the classical twin study was established in the 1920s, the variety of traits and characteristics that were studied expanded, comprising a large number of psychological, educational, medical and physical variables. Not very much happened with the statistical methods, however, until path analysis and structural equation modelling were adopted from around 1975 and used to develop much more complex statistical models. Within a decade or two the focus had changed from estimating the heritability of single traits to longitudinal and multivariate modelling. Here quite new questions were asked. Do genes and environmental factors that influence a certain trait change throughout life? When certain traits - like anxiety disorders and depression, or like verbal, spatial and numerical intelligence - covary, is it because the same genes code for the different traits, or is it perhaps because the traits are influenced by the same environmental factors? New kinds of "extended" twin designs were developed, in which data from relatives of twins were included. Such designs give more precise estimates than do data from twins alone and permit other types of effects to be examined. For example, studies which include parents and siblings of the twins can split the family environment into effects common to parents and offspring, common to twins, and common to ordinary siblings. Likewise, they can estimate nonadditive genetic effect and environmental effect shared by twins in the same model, whereas these parameters are fully negatively confounded in the classical twin design. Non-random mating, that is, phenotypic (and genetic) spouse correlation, mimics effect of common environment if not corrected for in classical twin studies, whereas extended studies permit both a direct estimation of the spouse correlation and the inclusion of it as a separate model parameter. The study by Heath et al. from 1985, reprinted in this issue, is an example of an extended twin study, including twins and the parents of the twins. As discussed in this issue by one of the coauthors, Jon M. Sundet, this classical paper based on Norwegian data also shows how twin studies can be of interest to the social sciences.

The rapid development of method advancements during the eighties and nineties continued into the new century, but now at a somewhat slower pace. Rather than the presentations of groundbreaking new methods, the last one or two decenniums have seen an extensive body of results highly relevant to medicine, psychology and social sciences. Most of this research uses methods developed during the late twentieth century. According to a recent meta-analysis by Polderman et al. (described by Røysamb and Tambs and by Nilsen et al. in this special issue), intending to include all twin studies during the last 50 years, the mean number of yearly published twin studies internationally was 7 in the sixties, 18 in the seventies, 60 in the nineties, and 145 in the period 2000-2012 (data showed in Polderman et al., Supplementary Table 2). Mean sample size was 104 in the seventies, 385 in the eighties, 634 in the nineties, and 995 from year 2000 on. This expansion is more than anything accounted for by much better twin registries, better education on twin methodology, better and more specialized computer software, and much more powerful hardware, which have lowered the threshold for a new generation to start a career in twin research and quantitative genetics worldwide. From struggling with accusations of scientific fraud from extreme environmentalist social scientists before the millennium, twin research has now been recognized as a powerful device in genetic epidemiology. Most scientific psychological or medical journals, highly specialized as well as general, now welcome twin research. Even in the shade of molecular genetics, often considered the scientific revolution of the 21 century, traditional quantitative genetics thrives and flourishes.

The Nordic countries, with their early established high quality population registries, have had special advantages in the building of big population based twins panels. Norway's twin registry, as described by Nilsen et al. in this issue, is among the relatively few around the world based on public registry information and therefore among the most representative of the total population. According to a listing of 70 twin registries worldwide by Hur and Craig (Twin Research and $\mathrm{Hu}-$ man Genetics, 2013; 16: 1-12) the Norwegian registry is also the twelfth largest. Still it cannot fully match those from Sweden, Denmark and Finland, which were established earlier and have had better National funding. Perhaps the legal regulations have also been more restrictive on Norway. Such regulations in Nor- 
way recently required that twins voluntarily enrolled in previous twin studies had to be excluded from the twin registry unless they responded to a new informed consent form, which substantially reduced the number of twin pairs in the registry born after 1966. Although last in the Nordic class, Norway has successfully contributed to international twin research since the famous psychiatric studies in the late 1960s by Einar Kringlen, who also establish the first Norwegian twin registry. The registry was extended by Kåre Berg and Per Magnus at the University of Oslo in the 1970s. Magnus initiated the set-up of the youngest part of the twin registry at the Norwegian Institute of Public Health in 1992, including twins born 1967 or later. He was also taking the initiative to merging the different registries into a single registry, The Norwegian Twin Registry, in 2009. In the present issue of Norsk Epidemiologi Magnus et al. describe the establishments of the various Norwegian twin panels which are today part of the Norwegian Twin Registry. They also give an overview of early and more recent research based on twin registry data. In the aforementioned metaanalysis by Polderman et al. (Supplementary Table 3), Norway is ranked eighth in the world in number of published twin studies.

Worldwide the participation rates for questionnaire studies are declining. Even though twin studies usually benefit from better participation than other questionnaire or interview studies, decreasing attendance is a problem in twin studies as well, and Norway is no exception. For the future the strategy must be to invest a maximum effort into the recruitment of as many twins as possible to the twin registry and to link data from the twin registry with data from other public registries. Considering a low expected future participation rate, perhaps less should be invested in further collections of questionnaire data. Norway, with its high quality registries, will have an international advantage in future twin studies on registry data.

Does traditional quantitative genetics, using data from twin, family and adoption studies, have a future alongside molecular genetics, or will the latter in due time be able to answer all the important questions alone? We think twin studies are here to stay for new centuries. "Complex" diseases, psychological traits, and other individual characteristics have typically proved to be influenced by a very large number of segregating genes, each with correspondingly small effects. Usually single genes shown to code for a trait can only explain up till a few percent of the phenotypic variance. But even if a set of specific genes once in the future, for some complex phenotypes, could be shown to account for the majority of the phenotypic variance, molecular studies are not informative about environmental effects. Only twin studies can estimate the total environmental effects and then split the environmental effect into shared and non-shared effects. And only extended twin or family studies can estimate environmental family effects specific to different types of first degree relatives.

The purpose of this special issue is to give the reader a glance of previous and current twin studies in Norway. All key researchers have been invited to contribute, and although the final content do not cover all ongoing studies, it shows the importance of quantitative genetic methods in elucidating very different topics - from personality via social benefits to arthrosis. Hopefully the content of the issue illustrates the vitality and potential of Norwegian twin research and of twin research in general. The society should more highly appreciate this research, and more than anything we must all be grateful to the tens of thousands of Norwegian twins who have taken part in the various studies and given of their time to help science.

\section{The guest editors}

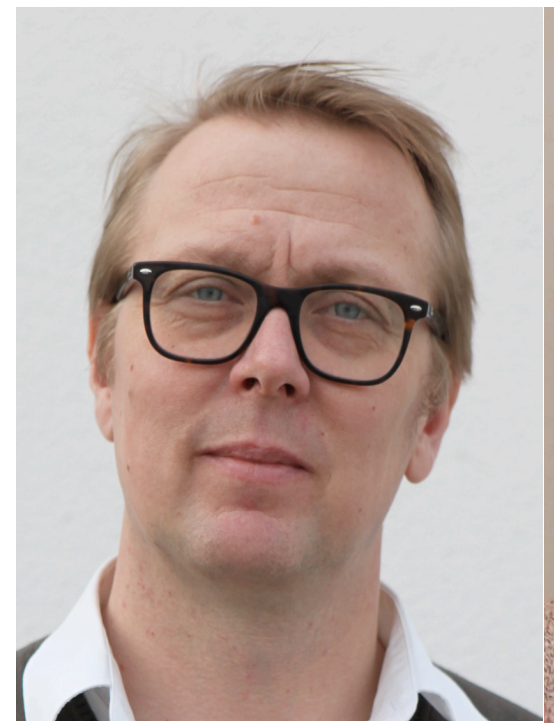

Thomas Sevenius Nilsen

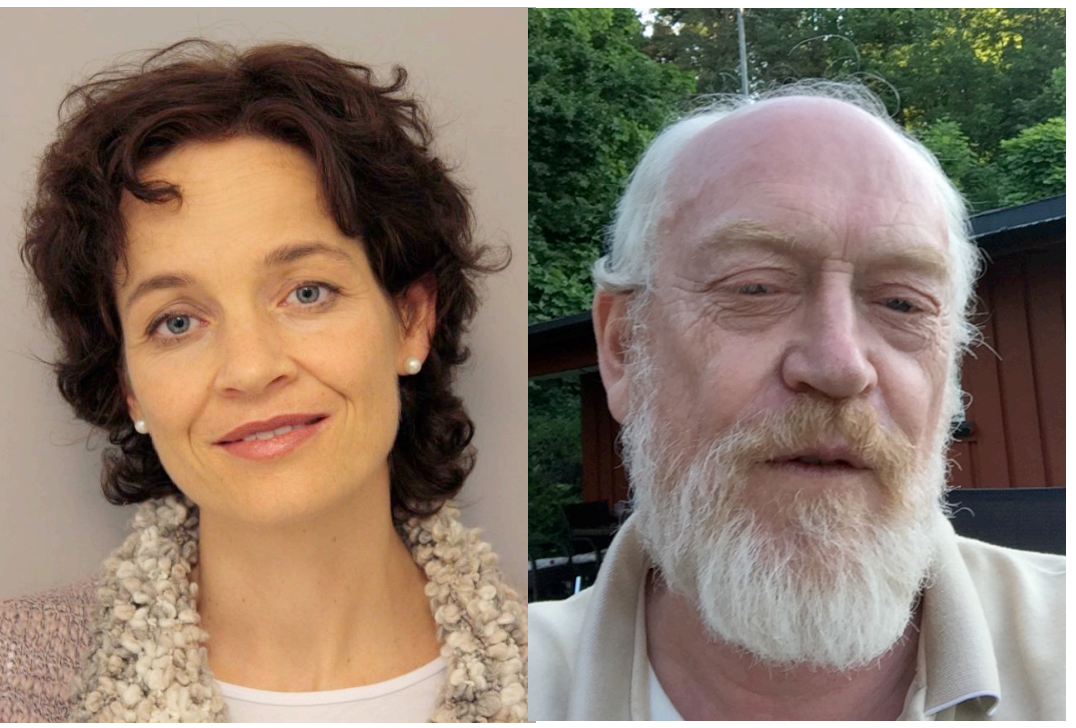

Ragnhild Elise Ørstavik
Kristian Tambs 